\title{
Experiment Development of Control Point Positioning Based on RTK
}

\author{
Shao Mingqin ${ }^{1,}$,, Yang Xuelin ${ }^{2, b}$ \\ 1Maritime College of Shandong Jiaotong University Weihai, China \\ 2Beijing aerospace University Beijing, Chain
}

Key words: experiment, control point positioning, RTK, accuracy analysis

\begin{abstract}
This paper has a detailed introduce on parts of differential GPS system and construction method in control point positioning experiment .This paper also solves some problems, such as excluded from multipath interference. This paper focuses on analyzing the result accuracy of RTK positioning method. This experiment can eventually achieve the positioning accuracy is 2 centimeters or so.
\end{abstract}

\section{Obtain Standard coordinates of base station \& control point and select the experimental field}

Before the start of the experiment, put the GPS receiver in the base station and the control point, use the GPS single point positioning method for positioning, get a set of positioning information every one second and lasted one hour. Research shows that the error of single point GPS positioning obeys Gauss distribution, calculate the average value of all the obtained positioning information, this average value can be considered as the exact coordinate information of the measured position. The error judgment can be carried out by comparing the results of differential GPS positioning with the obtained exact coordinate information in the following experiment.

In the experiment operation, in order to improve the reliability of base station data and ensure that the base station and the user station can receive more and the same satellite signal at the same time, the base station is located in a higher position generally and there is no obstruction all around. In the process of experiment, positioning experiments were carried out respectively many times at four positioning points in different height and different obstruction. (This four positioning points are: the roof platform of the second floor, the roof platform of the sixth floor, the courtyard hanging ladder of the sixth floor and the roof platform of the eleventh floor in the new main building) Compared with experimental data at this four positioning points, about 17 satellites positioning signal can be received at the roof platform of the eleventh floor in the new main building, there are many signals in this positioning point, so the reliability of the experimental result is the highest. In addition, this position is open around and there is no shielding effect of the surrounding buildings, therefore multipath effect errors can be effectively avoided. So select the eleventh roof platform of the new main building as the formal experimental field and carry out the control point positioning experiment.

\section{Construction of experimental equipment and positioning system}

A base station and a receiving station need to be built in the positioning experiment and need to establish a wireless transmission data link between them.

The receiver that is to be used for both the base station and the user station are the FelxPak-G2-V1 device of the NovAtel OEMV series, these two devices have the same performance.

Digi XTend-PKG wireless modem is used for the establishment of the wireless transmission data link, it is equivalent to establish a pair of wireless serial port between the base station and the user station. [2]

The wiring diagram of differential positioning is shown in figure 1: 


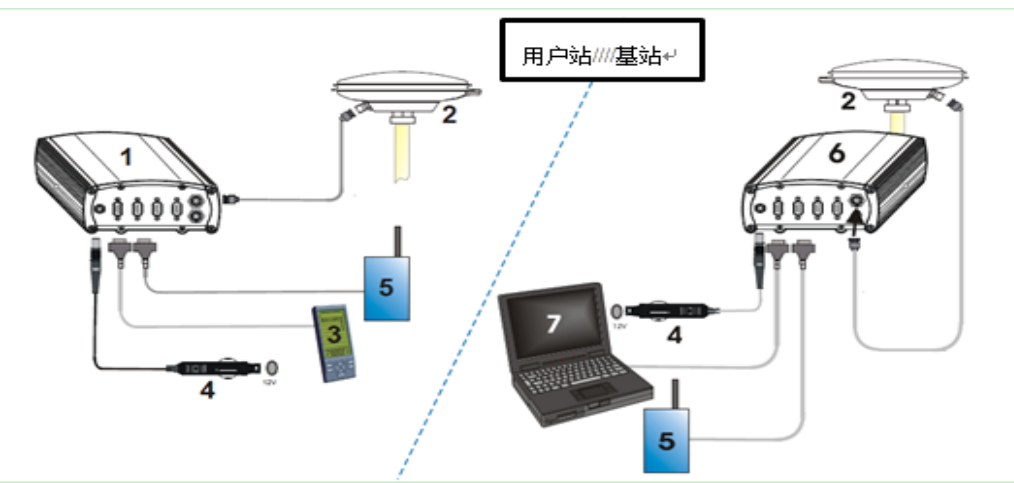

Figure 1, differential GPS operation wiring diagram

The meaning of each number in this figure is:

1. GPS receiver of the user station 2. GPS receiving antenna 3. Device provided by user for storing data in a COM1 port 4 . Power supply provided by user 5 . Radio station equipment provided by user, it is connected to com 2 port 6 . GPS receiver of the base station 7 . Computer provided by user, it is connected to com1 port, it is used for starting GPS receiver and monitoring the working status of GPS receiver

\section{Experimental operation}

1. Build the differential system according to the wiring diagram, supply power to the GPS receiver and radio station by using its own power supply. Before carrying out differential GPS positioning experiment, testing the effective range of the wireless data transmission and the corresponding baud rate that can transmit data correctly by using the software which is provided by Digi company, ensure that the base station \& the user station can work in this effective range and sending and receiving information by using the corresponding baud rate.

2. Open the monitoring software of the receiver on the computer, the computer can be connected to the base station and the user station respectively through the USB interface or serial port, Observe the status of Solution Type in Position Window, if it is single and the Column of Solution Status displays computed, it proves the receiver works in the normal single station positioning mode at this time.

3. Standard coordinates of base station and user station are obtained by mean of calculating the average value, Calculate the average value by using the instruction of the monitoring software. After reach the setting time, Observe the status of Solution Type in Position Window, if it is fixed and the displayed longitude, latitude and height is stable, it proves that the calculation for average value is successful, so the displayed result is the average value.

4. Send the related instruction to the base station and the user station respectively, enter the differential GPS positioning mode. The related instructions are as follows:

5. Observe the positioning result and record the data

In the process of positioning, the data update frequency of CDU is 1 time per second, manual input data can't keep up with the update speed of the system, under the Tools menu select Logging Control Window, then complete the relevant settings for data storage in the open window (the main setting is the format of the data in the storage file), store the positioning results in the file of ".gps" type, after the end of storage, open this file use txt to get all of the positioning data.

\section{Experimental results and accuracy analysis}

1. Obtain the standard coordinates of base station and user station

Standard coordinates of base station and user station are obtained by mean of calculating the average value of single point positioning within a certain period of time (take 1 hour in the experiment), after completing the calculation, Position Window interface is shown in figure 2: 


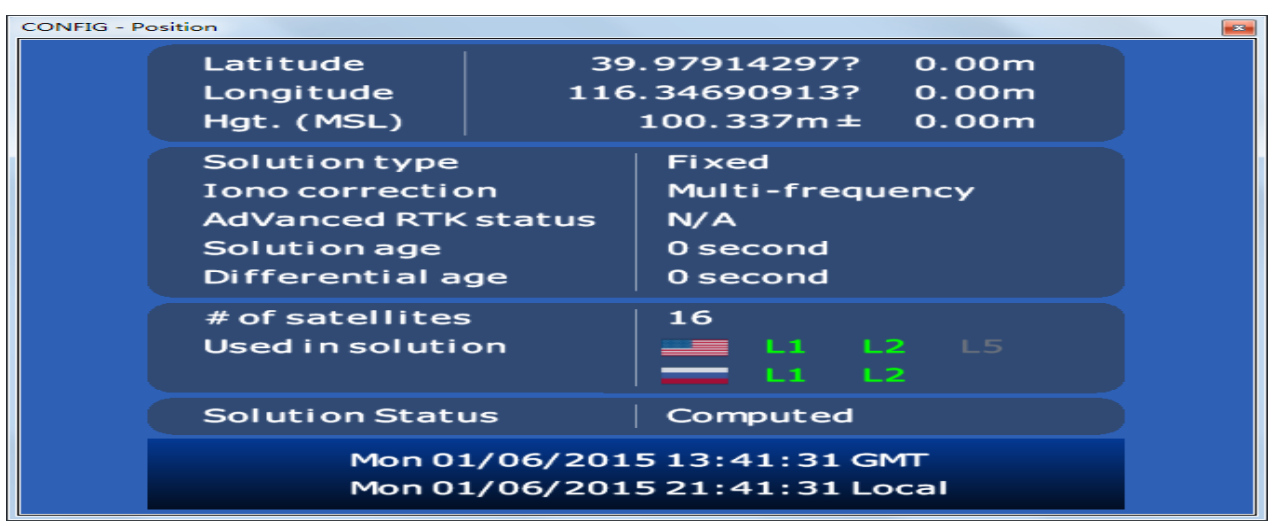

Figure 2, interface of calculating average value

The data obtained is shown in table 1 :

Table 1, standard coordinates of base station and user station

\begin{tabular}{|c|c|c|c|}
\hline & Latitude(degree) & Longitude(degree) & Height(m) \\
\hline $\begin{array}{c}\text { Standard coordinate of } \\
\text { base station }\end{array}$ & 39.97914297 & 116.34690913 & 100.337 \\
\hline $\begin{array}{c}\text { Standard coordinate of } \\
\text { user station }\end{array}$ & 39.97914631 & 116.34690885 & 100.327 \\
\hline
\end{tabular}

Convert the difference of coordinates for base station and user station into distance, this distance is $0.372 \mathrm{~m}$. The measured distance (using steel tape) between them is 0.356 meters, so the difference between the measured distance and the actual distance is $0.016 \mathrm{~m}$. This error is within acceptable limits.

2. Obtain differential coordinates of user station and analysis accuracy in RTK mode $[3,4]$

Use the average coordinate of the base station as standard coordinate to calculate differential correction, then obtain the differential result for user station, at this time the Position Window interface is shown in figure 3:

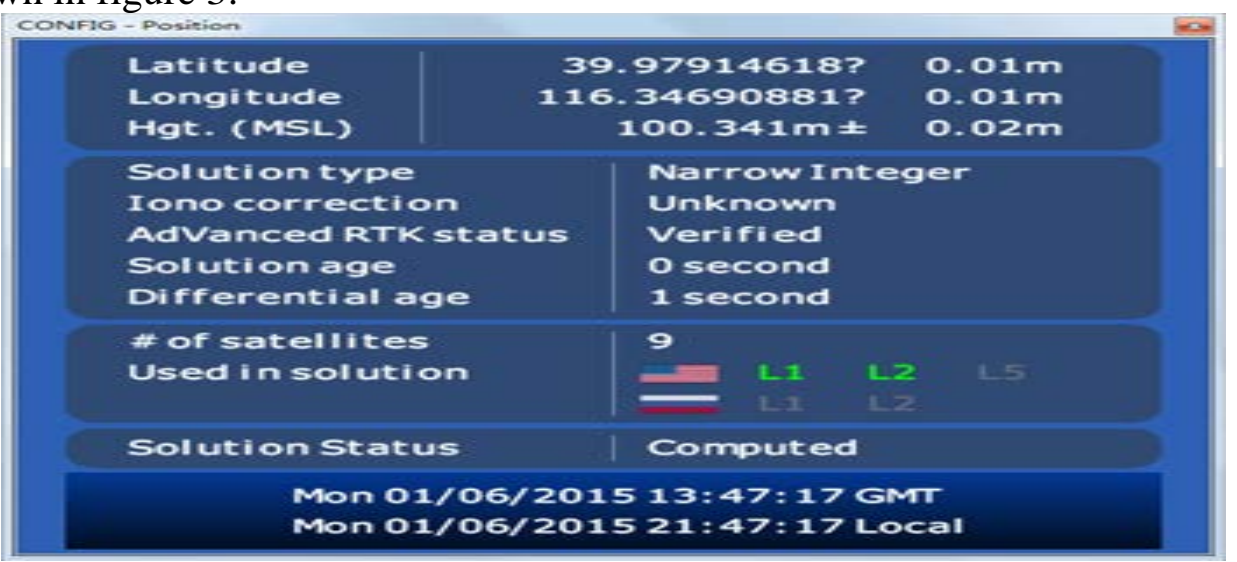

Figure 3, differential RTK interface

The solution type column of user station interface change from Fixed to Narrow Integer, the measured data of longitude, latitude and height is constantly updated and the estimated accuracy of this system is automatically given behind the data, this shows that the system has been correctly working in RTK mode. Compared the results of the RTK in 20 seconds with the average coordinate of the user station (it is considered as standard coordinates for user station) which is previously measured, the obtained data is shown in table 2:

Table 2, RTK accuracy analysis table

\begin{tabular}{|c|c|c|c|c|}
\hline \multicolumn{5}{|c|}{ RTK differential GPS accuracy analysis table (Narrow Integer) } \\
\hline & $\begin{array}{c}\text { Latitude } \\
\text { (degree) }\end{array}$ & $\begin{array}{r}\text { Longitude } \\
\text { (degree) }\end{array}$ & $\begin{array}{r}\text { Height } \\
\text { (m) }\end{array}$ & $\begin{array}{c}\text { Distance from } \\
\text { user station(m) }\end{array}$ \\
\hline Standard coordinate of user station & 39.97914631 & 116.34690885 & 100.327 & 0.0000 \\
\hline RTK positioning coordinate 1 & 39.97914620 & 116.34690881 & 100.343 & 0.0204 \\
\hline RTK positioning coordinate 2 & 39.97914621 & 116.34690884 & 100.338 & 0.0157 \\
\hline
\end{tabular}




\begin{tabular}{|c|l|l|l|l|}
\hline RTK positioning coordinate 3 & 39.97914619 & 116.34690881 & 100.342 & 0.0204 \\
\hline RTK positioning coordinate 4 & 39.97914616 & 116.34690882 & 100.341 & 0.0219 \\
\hline RTK positioning coordinate 5 & 39.97914619 & 116.34690880 & 100.340 & 0.0191 \\
\hline RTK positioning coordinate 6 & 39.97914618 & 116.34690883 & 100.342 & 0.0209 \\
\hline RTK positioning coordinate 7 & 39.97914620 & 116.34690882 & 100.341 & 0.0188 \\
\hline RTK positioning coordinate 8 & 39.97914617 & 116.34690884 & 100.343 & 0.0223 \\
\hline Average distance & & & & 0.0199 \\
\hline
\end{tabular}

Observe the data in this table, the maximum distance difference between the RTK positioning coordinate of the user station and the standard coordinate of the user station is no more than $2.3 \mathrm{~cm}$, in addition, considered that the average distance difference within 20 seconds may less than $2 \mathrm{~cm}$, If we continue to carry out RTK positioning, positioning accuracy can be improved on the basis of 2.3 $\mathrm{cm}$ by calculating the average value.

Data in this table shows that the height difference between the RTK positioning coordinate and the standard coordinate is large, this indicates that the height error is more obvious in positioning errors.

\section{References:}

[1]Qiang Liu. Design of differential GPS moving target tracking and positioning system [D]. Shanxi: Zhongbei University, 2013. 1-24.

[2]Zhou Xu. Study on GPS differential positioning technology and implementation method [D]. Henan: The PLA Information Engineering University, 2006.

[3]Tianwen Li. GPS principle and application (Third Edition) [M]. Beijing: Science Press, 2015:4-14.

[4]Jie Yang, Fan Zhang. Comparative study on high precision GPS differential positioning technology [J]. Mobile communication, 2014, (2): 54-58.

[5]Yi Jing. and implementation of data mining technology in the hospital information [j]. Chongqing University of medical sciences 2007.

[6]Kantardzic M. Data Mining Concept、Models、Methods and Algorithms[M]. IEEE Press.2002 